\title{
Pest regulation by means of impulsive controls
}

\author{
Paul Georgescu *, Gheorghe Moroşanu \\ Department of Mathematics and Its Applications, Central European University, Nador u. 9, 1051 Budapest, Hungary
}

\begin{abstract}
In this paper, we consider an integrated pest management model which is impulsively controlled by means of biological and chemical controls. These controls are assumed to act in a periodic fashion, a nonlinear incidence rate being used to account for the dynamics of the disease caused by the application of the biological control. The Floquet theory for impulsive ordinary differential equations is employed to obtain a condition in terms of an inequality involving the total action of the nonlinear force of infection in a period, under which the susceptible pest-eradication solution is globally asymptotically stable. If the opposite inequality is satisfied, then it is shown that the system under consideration becomes uniformly persistent. A biological interpretation of the persistence condition is also provided.
\end{abstract}

(c) 2007 Elsevier Inc. All rights reserved.

Keywords: Pest management; Impulsive controls; Nonlinear infection rate; Stability; Persistence

\section{Introduction}

Lately, it has been widely noticed that pesticide abuse has unwelcomed long-term environmental consequences and ultimately damages human health. In some instances, the persistent use of pesticides has increased the incidence of pest varieties which are resistant to chemicals, rendering chemical controls almost ineffective, while in other situations the use of such products changed for the worse the balance between the beneficial and the harmful insects. Consequently, sophisticated and multifaceted ecosystem-based strategies have been constructed in order to minimize the use of hazardous chemicals.

Integrated pest management (IPM) is an ecological approach which represents a synthesis of techniques of various natures to control pests, with an accent on those which are potentially less damaging to the environment. Further, techniques specifically suited to the target pests are preferred, in order to avoid harmful effects on non-target organisms. Specifically, natural predators, parasites or pathogens of pests may be used, together with genetically-engineered pest-resistant varieties of crops, mechanical methods of pest control such as traps or insect barriers and habitat manipulation. In this approach, pesticides are used only as a last resort, when deemed an absolute necessity. For a historical perspective on the evolution of IPM definitions, see [2].

\footnotetext{
${ }^{*}$ Corresponding author.

E-mail addresses: vpgeo@go.com (P. Georgescu), Morosanug@ceu.hu (G. Moroşanu).
} 
The purpose of IPM is often to drive the size of the pest population under certain economically significant levels, not to eradicate it totally, as the latter can be impossible, cost-ineffective or potentially damaging to the environment.

In this regard, the economic injury level (EIL), as defined in [17], is the lowest population density of a pest that will cause economic damage, or the amount of pest injury which will justify the cost of using controls. Another related type of injury level which can be of interest in certain situations is called the aesthetic injury level (AIL) and is defined in the same manner, but based on aesthetic rather than economic considerations. Finally, a relevant parameter is also the action threshold, representing the pest density at which control measures should be implemented in order to prevent the pest populations to reach the economic (or aesthetic) injury level. See $[12-14,17]$ for further details.

Chemical control relies mainly on the use of synthetic pesticides to suppress pests. Biological pesticides, derived from plants or microorganisms, such as Bacillus thuringiensis are also of use in chemical control.

Biological control is defined as the reduction of pest populations by using their natural enemies (see Hoffmann and Frodsham [5]). An approach to control insect pests, for instance, is to release parasitoids or pathogens. While the first are generally species which develop within or on the host and ultimately kill it, the latter are viruses, fungi or bacteria which kill or incapacitate the host by causing a disease, or otherwise affect the biological processes within the host. Another biological approach to pest control is to release pests which are infected in laboratories, with the purpose to maintain the endemicity of the disease in the target pest population, as infected pests generally cause less environmental damage. Usually, biological controls are low-cost and have minimal negative environmental impact.

Regarding disease transmission, in many papers on epidemiological models treating disease dynamics in animal populations, use is made of an incidence rate of infection which is bilinear in both the susceptible and the infective fraction of the total number of individuals. However, the assumption of homogeneous mixing, which motivates the mass action law behind the use of the bilinear incidence rate, may not be accurate under certain conditions. This is the case, for instance, when the concentration of infective pests is very high and saturation may occur or if multiple exposure to the disease vector or multiple contacts are required for disease transmission.

Also, many classical models using bilinear incidence rates exhibit threshold dynamics, that is, if the so-called basic reproduction number $R_{0}$ (that is, the average number of new infections produced by a single infective individual introduced in a totally susceptible population) is greater than 1 , then the disease remains endemic and the endemic equilibrium is globally asymptotically stable, while if $R_{0}$ is lower than 1 , then the disease dies out, the endemic equilibrium loses its stability and and the disease-free equilibrium becomes globally asymptotically stable. In this setting, the dynamics of the system is unaffected by the relative sizes of the initial populations. However, it has been observed that many diseases exhibit yearly variations in a periodic fashion and that for some diseases the persistence of the infective populations depends on their initial sizes. See Wang [18] for a more detailed discussion regarding this matter, which also outlines the fact that the nonlinearity of the force of infection (that is, the per-susceptible rate of infection, sometimes named the attack rate, which is generally understood as a function of the total number of infective individuals) may appear as a result of intervention policies.

Liu et al. [9] studied a SEIRS model with nonlinear incidence rates of type $\lambda I^{p} S^{q}, 0<p \leqslant 1,0<q \leqslant 1$, where $I$ denotes the size of the infective population and $S$ denotes the size of the susceptible population, and observed that while the choice of a $q \neq 1$ does not have a decisive impact on the qualitative behavior of the system, the choice of a $p \neq 1$ modifies the phase portrait of the system in an obvious manner. In the latter case, the meaning of the basic reproduction number understood as a threshold parameter for the stability of the system vanishes completely, since the disease remains endemic and the system always approaches a unique endemic equilibrium irrespective of $q$.

An incidence rate of type $g(I) S$ has been proposed by Capasso and Serio in [3], with $g(I)=k I /(1+\alpha I)$. This incidence rate includes behavioral change and crowding effect for infectives. A general incidence rate of type $g(I) S$ with $g(I)=k I^{p} /\left(1+\alpha I^{q}\right)$ has been employed by Liu et al. in [10]; see also Hethcote and van den Driessche [4]. Particular incidence rates of type $g(I) S=k I^{2} S /\left(1+\alpha I^{2}\right), g(I) S=K I S /\left(1+\alpha I^{2}\right)$ have been used in Ruan and Wang [15], respectively, in Xiao and Ruan [19]. 
Models with general nonlinear incidence rates of type $g(I) h(S)$ and $f(I, S)$ have been treated in Korobeinikov and Maini [7] and in Korobeinikov [6], respectively, and it has again been observed that the dependence on $I$ plays a more prominent role in the stability of the endemic equilibrium than the dependence on $S$.

In the following, we shall use a general incidence rate of type $g(I) S$ to model disease transmission, under a few biologically feasible conditions on the nonlinear force of infection $g$.

The purpose of this paper is to construct a model of pest control using both biological controls (periodic release of pests which are infected in laboratories) and chemical controls (pesticide spraying). To account for the discontinuity of the human activities (that is, pesticides are usually not sprayed all year round, but at fixed moments of the year), we propose a model in which both controls are used in an impulsive and periodic fashion, with the same period but not in the same time.

This paper is organized as follows: in Section 2, we formulate the main biological assumptions used in the paper and subsequently employ them to construct our impulsive control model. In Section 3, we introduce a number of definitions and state a few basic auxiliary results. In Section 4, we study the permanence of our system. Finally, we conclude in Section 6 with a brief discussion of our main findings.

\section{The model}

In the following, we denote by $S$ the size of the susceptible pest population, by $I$ the size of the infective pest population, and suppose that all pests are either susceptible or infective. To formulate our mathematical model, we rely on the following biological assumptions.

(A1) The intrinsic growth rate of the susceptible pest population in the absence of infection is given by the nonlinear function $\operatorname{Sn}(S)$, where $n$ satisfies certain assumptions outlined below.

(A2) The infective pests neither recover nor reproduce.

(A3) The infective pests neither damage crops nor contribute to the total size of the environment-supported population.

(A4) The incidence rate of the infection is nonlinear in $I$ and given by $g(I) S$, where $g$ satisfies certain assumptions outlined below.

(A5) Infected pests are released in an impulsive and periodic fashion, in a fixed amount $\mu$ each time.

(A6) Pesticides are sprayed in an impulsive and periodic fashion, with the same period as the action of releasing infective pests but at different moments. As a result, fixed proportions $p_{1}$ and $p_{2}$ of susceptible pests and infective pests, respectively, are killed each time.

On the basis of the above assumptions, we may formulate the following impulsively controlled model which characterizes the behavior of the system under consideration.

$$
\begin{cases}S^{\prime}(t)=S(t) n(S(t))-g(I(t)) S(t), & t \neq(n+l-1) T, t \neq n T ; \\ I^{\prime}(t)=g(I(t)) S(t)-w I(t), & t \neq(n+l-1) T, t \neq n T ; \\ \Delta S(t)=-\delta_{1} S(t), & t=(n+l-1) T \\ \Delta I(t)=-\delta_{2} I(t), & t=(n+l-1) T \\ \Delta S(t)=0, & t=n T \\ \Delta I(t)=\mu, & t=n T .\end{cases}
$$

Here, $T>0,0<l<1, \Delta \varphi(t)=\varphi(t+)-\varphi(t)$ for $\varphi \in\{S, I\}, 0 \leqslant \delta_{1}, \delta_{2}<1, n \in \mathbb{N}^{*}$.

The functions $n$ and $g$ satisfy the following hypotheses indicated below.

(N) $n(0)=0, n$ is decreasing on $[0, \infty), \lim _{S \rightarrow \infty} n(S)<-w, S \mapsto \operatorname{Sn}(S)$ locally Lipschitz on $(0, \infty)$.

(G) $g(0)=0, g$ is increasing and globally Lipschitz on $[0, \infty)$.

Note that the hypothesis $(\mathrm{G})$ is verified for three commonly used forces of infection, namely for $g_{1}(x)=a x$, $g_{2}(x)=\frac{a x}{1+m x}, g_{3}(x)=k\left(1-\mathrm{e}^{-a x}\right)$, as they are strictly increasing on $[0, \infty)$ and are globally Lipschitz functions 
since their derivatives are uniformly bounded on $[0, \infty)$. Also, hypothesis $(\mathrm{N})$ is satisfied if the intrinsic growth rate of the susceptible population $\operatorname{Sn}(S)$ is given by the logistic growth law $(\operatorname{Sn}(S)=r S(1-S / K))$ or by Gompertz's growth law $(S n(S)=r S \ln (K / S))$. In what follows, let us denote $n(0)=r$. Let us also observe that, in view of $(\mathrm{G}), g(x) \leqslant L x$ for $x \geqslant 0$, where by $L$ we denote the Lipschitz constant of $g$.

Models related to ours have been studied by Zhang and Chen in [20], where the intrinsic growth rate of the susceptible population is of logistic type, which corresponds to $n(S)=1-S / K$, but a particular incidence rate of type $I h(S)$ is used rather than of type $g(I) S$, and by Liu et al. in [8], where an impulsively controlled system which models the dynamics of a prey-dependent consumption model is studied by similar methods. See also Song and Xiang [16] where an IPM strategy for a two-prey one-predator model with stage structure for predator is described, or Liu et al. [11], where the impulsive controllability of a predator-prey system with Ivlev functional response is studied. Note that, from a formal point of view, the above-mentioned predator-prey models are related to our model.

It is also to be noted that our model can accommodate some situations in which the infective pest population contributes to the growth of the total population size towards the carrying capacity of the environment, that is, when (A3) is only partially satisfied. Precisely, if the first equation in our model is substituted by

$$
S^{\prime}(t)=S(t)\left(1-\frac{S(t)+I(t)}{K}\right)-g(I(t)) S(t)
$$

then this equation can be rearranged as

$$
S^{\prime}(t)=S(t)\left(1-\frac{S(t)}{K}\right)-\left(g(I(t))+\frac{I(t)}{K}\right) S(t)
$$

which again fits our framework. Moreover, the decreases in the number of susceptible and, respectively, of infective pests in the third and fourth equation of (S) can also be achieved through selective catching rather than by pesticide poisoning only.

Under these assumptions, it is seen that the Cauchy problem for the system (S) has a unique positive and global solution for positive initial data $(S(0), I(0))$, while if the initial data is strictly positive, then the corresponding solution is also unique, strictly positive and global, that is, the Cauchy problem for (S) with positive initial data is biologically well-posed.

\section{Preliminaries}

In this section, we shall introduce some definitions and notations and state a few basic results which will be useful in what follows.

Let us denote by $f=\left(f_{1}, f_{2}\right)$ the mapping defined by the right-hand side of (S). Let also $\mathscr{V}_{0}$ be the set of functions $V: \mathbb{R}_{+} \times \mathbb{R}_{+}^{2} \rightarrow \mathbb{R}_{+}$which are locally Lipschitz in the second variable, continuous on $((n+l-1) T, n T] \times \mathbb{R}_{+}^{2}$ and on $(n T,(n+l) T] \times \mathbb{R}_{+}^{2}$ and for which the limits $\lim _{(t, y) \rightarrow((n+l-1) T+, x)} V(t, y)=$ $V((n+l-1) T+, x)$ and $\lim _{(t, y) \rightarrow(N T+, x)} V(t, y)=V(n T+, x)$ exist and are finite for $x \in \mathbb{R}_{+}^{2}$ and $n \in \mathbb{N}^{*}$.

For $V \in \mathscr{V}_{0}$, we define the upper right Dini derivative of $V$ with respect to the system (S) at $(t, x) \in((n+l-1) T, n T) \times \mathbb{R}_{+}^{2}$ or $(n T,(n+l) T) \times \mathbb{R}_{+}^{2}$ by

$$
D^{+} V(t, x)=\lim \sup _{h \downarrow 0} \frac{1}{h}[V(t+h, x+h f(t, x))-V(t, x)] .
$$

We now indicate a comparison result for solutions of impulsive differential inequalities which allows us to estimate the values of the solutions of (S). We suppose that $h: \mathbb{R}_{+} \times \mathbb{R}_{+} \rightarrow \mathbb{R}$ satisfies the following hypotheses.

(H) $h$ is continuous on $((n+l-1) T, n T] \times \mathbb{R}_{+}$and on $(n T,(n+l) T] \times \mathbb{R}_{+}$and the limits $\lim _{(t, y) \rightarrow((n+l-1) T+, x)}$ $h(t, y)=h((n+l-1) T+, x), \lim _{(t, y) \rightarrow(N T+, x)} h(t, y)=h(n T+, x)$ exist and are finite for $x \in \mathbb{R}_{+}$and $n \in \mathbb{N}^{*}$. 
Lemma 3.1 ([1]). Let $V \in \mathscr{V}_{0}$ and assume that

$$
\begin{cases}D^{+} V(t, x(t)) \leqslant h(t, V(t, x(t))), & t \neq(n+l-1) T, n T \\ V(t, x(t+)) \leqslant \psi_{n}^{1}(V(t, x(t))), & t=(n+l-1) T \\ V\left(t, x(t+) \leqslant \psi_{n}^{2}(V(t, x(t))),\right. & t=n T\end{cases}
$$

where $h: \mathbb{R}_{+} \times \mathbb{R}_{+} \rightarrow \mathbb{R}$ satisfies $(H)$ and $\psi_{n}^{1}, \psi_{n}^{2}: \mathbb{R}_{+} \rightarrow \mathbb{R}_{+}$are non-decreasing for all $n \in \mathbb{N}$. Let $r(t)$ be the maximal solution of the scalar impulsive differential equation

$$
\begin{cases}u^{\prime}(t)=h(t, u(t)), & t \neq(n+l-1) T, n T ; \\ u(t+)=\psi_{n}^{1}(u(t)), & t=(n+l-1) T ; \\ u(t+)=\psi_{n}^{2}(u(t)), & t=n T ; \\ u(0+)=u_{0} & \end{cases}
$$

defined on $[0, \infty)$. Then $V\left(0+, x_{0}\right) \leqslant u_{0}$ implies that $V(t, x(t)) \leqslant r(t)$ for all $t \geqslant 0$, where $x(t)$ is an arbitrary solution of (3.1).

Note that the maximality property used in Lemma 3.1 refers to the maximality of values rather than to the maximality of the domain and that under appropriate regularity conditions (3.2) has a unique solution. In that case $r$ becomes the unique solution of (3.2). We now indicate a result which provides an estimation for the solution of a system of differential inequalities.

Lemma 3.2 ([1]). Let the function $u \in P C^{1}\left(\mathbb{R}_{+}, \mathbb{R}\right)$ satisfy the inequalities

$$
\left\{\begin{array}{l}
\frac{\mathrm{d} u}{\mathrm{~d} t} \leqslant p(t) u(t)+f(t), \quad t \neq \tau_{k}, t>0 ; \\
u\left(\tau_{k}+\right) \leqslant d_{k} u\left(\tau_{k}\right)+h_{k}, \quad k \geqslant 0 \\
u(0+) \leqslant u_{0},
\end{array}\right.
$$

where $p, f \in P C\left(\mathbb{R}_{+}, \mathbb{R}\right)$ and $d_{k} \geqslant 0, h_{k}$ and $u_{0}$ are constants and $\left(\tau_{k}\right)_{k \geqslant 0}$ is a strictly increasing sequence of positive real numbers. Then, for $t>0$,

$$
u(t) \leqslant u_{0}\left(\prod_{0<\tau_{k}<t} d_{k}\right) \mathrm{e}^{\int_{0}^{t} p(s) \mathrm{d} s}+\int_{0}^{t}\left(\prod_{0 \leqslant \tau_{k}<t} d_{k}\right) \mathrm{e}^{\int_{s}^{t} p(\tau) \mathrm{d} \tau} f(s) \mathrm{d} s+\sum_{0<\tau_{k}<t}\left(\prod_{\tau_{k}<\tau_{j}<t} d_{j}\right) \mathrm{e}^{\int_{\tau_{k}}^{t} p(\tau) \mathrm{d} \tau} h_{k} .
$$

In the above, by $P C\left(\mathbb{R}_{+}, \mathbb{R}\right)\left(P C^{1}\left(\mathbb{R}_{+}, \mathbb{R}\right)\right)$ is meant the class of real piecewise continuous (real piecewise continuously differentiable) functions defined on $[0, \infty)$. For other results on impulsive differential equations, see Bainov and Simeonov [1]. Using the above Lemma, it is now possible to prove that all solutions of (S) are bounded.

Lemma 3.3. There is $M>0$ such that $S(t) \leqslant M, I(t) \leqslant M$ for $t \geqslant 0$.

Proof. Let us define $u: \mathbb{R}_{+} \rightarrow \mathbb{R}_{+}$by

$$
u(t)=S(t)+I(t), \quad t>0 .
$$

Then

$$
\frac{\mathrm{d} u}{\mathrm{~d} t}+w u=S(n(S)+w), \quad t>0, \quad t \neq(n+l-1) T, \quad t \neq n T .
$$

Since $\lim _{S \rightarrow \infty} n(S)<-w$, it follows that the right-hand side of (3.4) is bounded from above and consequently there is $C>0$ such that

$$
D^{+} u+w u \leqslant C, \quad t>0, \quad t \neq(n+l-1) T, \quad t \neq n T .
$$

One also sees that

$$
u((n+l-1) T+) \leqslant(1-\delta) u((n+l-1) T)
$$


and

$$
u(n T+)=u(n T)+\mu,
$$

where $\delta=\min \left(\delta_{1}, \delta_{2}\right)$. It the follows from Lemma 3.2 that

$$
u(t) \leqslant u(0+)\left[\prod_{0<(n+l-1) T<t}(1-\delta)\right] \mathrm{e}^{-w t}+C \int_{0}^{t}\left[\prod_{s \leqslant(n+l-1) T<t}(1-\delta)\right] \mathrm{e}^{-w(t-s)} \mathrm{d} s+\sum_{0<n T<t} \mu \mathrm{e}^{-w(t-n T)}, \quad t>0,
$$

so

$$
u(t) \leqslant u(0+) \mathrm{e}^{-w t}+\frac{C\left(1-\mathrm{e}^{-w t}\right)}{w}+\mu \frac{\mathrm{e}^{w T}}{\mathrm{e}^{w T}-1}, t>0,
$$

and since the limit of the right-hand side of (3.6) as $t \rightarrow \infty$ is $C / w+\mu \mathrm{e}^{w T} /\left(\mathrm{e}^{w T}-1\right)$, it easily follows that $u$ is bounded on $[0, \infty)$.

We need now state a few basic results regarding the Floquet theory of impulsive ordinary differential equations. Let us consider the system

$$
\begin{cases}x^{\prime}(t)=A(t) x, & t \neq \tau_{k}, \quad t \in \mathbb{R} ; \\ \Delta x=B_{k} x, & t=\tau_{k}, \tau_{k}<\tau_{k+1}, \quad k \in \mathbb{Z},\end{cases}
$$

under the following hypotheses.

(H1) $A(\cdot) \in P C\left(\mathbb{R}, M_{n}(\mathbb{R})\right)$ and there is $T>0$ such that $A(t+T)=A(t)$ for all $t \geqslant 0$.

$(\mathrm{H} 2) B_{k} \in M_{n}(\mathbb{R}), \operatorname{det}\left(I_{n}+B_{k}\right) \neq 0$ for $k \in \mathbb{Z}$.

(H3) There is $q \in \mathbb{N}^{*}$ such that $B_{k+q}=B_{k}, \tau_{k+q}=\tau_{k}+T$ for $k \in \mathbb{Z}$.

Let $\Phi(t)$ be a fundamental matrix of (3.7). Then there is a unique nonsingular matrix $M \in M_{n}(\mathbb{R})$ such that $\Phi(t+T)=\Phi(t) M$ for all $t \in \mathbb{R}$, which is called the monodromy matrix of (3.7) corresponding to $\Phi$. Actually, all monodromy matrices of (3.7) are similar and consequently they have the same eigenvalues $\lambda_{1}, \lambda_{2}, \ldots, \lambda_{n}$, which are called the Floquet multipliers of (3.7). Under these hypotheses, the following result holds.

Lemma 3.4 ([1]). Suppose that conditions (H1)-(H3) hold. Then

1. The system (3.7) is stable if and only if all Floquet multipliers $\lambda_{k}, 1 \leqslant k \leqslant n$ satisfy $\left|\lambda_{k}\right| \leqslant 1$ and if $\left|\lambda_{k}\right|=1$, then to $\lambda_{k}$ there corresponds a simple elementary divisor.

2. The system (3.7) is asymptotically stable if and only if all Floquet multipliers $\lambda_{k}, 1 \leqslant k \leqslant n$ satisfy $\left|\lambda_{k}\right|<1$.

3. The system (3.7) is unstable if there is a Floquet multiplier $\lambda_{k}$ such that $\left|\lambda_{k}\right|>1$.

We now describe some properties of the subsystem

$$
\begin{cases}I^{\prime}(t)=-w I(t), & t \neq n T,(n+l-1) T \\ \Delta I(t)=-\delta_{2} I(t), & t=(n+l-1) T ; \\ \Delta I(t)=\mu, & t=n T ; \\ I(0+)=I_{0}, & \end{cases}
$$

which describes the dynamics of the susceptible pest eradication state. It will be seen that the system formed with the first three equations of (3.8) has a periodic solution to which all solutions of (3.8) tend as $t \rightarrow \infty$. We shall label this periodic solution with $I_{w}^{*}$, rather than, say, with $I_{w, \delta_{2}, \mu}^{*}$, as systems of type (3.8) occur throughout the paper for different $w^{\prime} s$ but always with the same $\delta_{2}$ and $\mu$ so there is no danger of confusion.

Lemma 3.5. The system constructed with the first three equations in (3.8) has a positive T-periodic solution $I_{w}^{*}$. With this notation, the following properties are satisfied. 
1. $\lim _{t \rightarrow \infty}\left|I(t)-I_{w}^{*}(t)\right|=0$ for all solutions $I(t)$ of (3.8).

2. $\sup _{t \geqslant 0}\left|I_{w_{1}}^{*}(t)-I_{w_{2}}^{*}(t)\right| \leqslant \mu C\left|w_{1}-w_{2}\right|$ for all $w_{1}, w_{2}>0$, where $C=C\left(w_{1}, w_{2}, \delta_{2}, T\right)$.

Proof. Obviously, one should have

$$
\begin{aligned}
& I_{w}^{*}(t)=\mathrm{e}^{-w t} I_{w}^{*}(0+), \quad t \in(0, l T] ; \\
& I_{w}^{*}(t)=\mathrm{e}^{-w t} I_{w}^{*}(0+)\left(1-\delta_{2}\right), \quad t \in(l T, T] .
\end{aligned}
$$

By the $T$-periodicity requirement, one obtains that

$$
\mathrm{e}^{-w T}\left(1-\delta_{2}\right) I_{w}^{*}(0+)+\mu=I_{w}^{*}(0+)
$$

so

$$
I_{w}^{*}(0+)=\frac{\mu}{1-\mathrm{e}^{-w T}\left(1-\delta_{2}\right)} .
$$

Consequently, the $T$-periodic solution $I_{w}^{*}$ of (3.8) is given by

$$
I_{w}^{*}(t)= \begin{cases}\frac{\mu \mathrm{e}^{-w(t-(n-1) T)}}{1-\mathrm{e}^{-w T}\left(1-\delta_{2}\right)}, & t \in((n-1) T,(n+l-1) T] \\ \frac{\mu \mathrm{e}^{-w(t-(n-1) T)}\left(1-\delta_{2}\right)}{1-\mathrm{e}^{-w T}\left(1-\delta_{2}\right)}, & t \in((n+l-1) T, n T] .\end{cases}
$$

Let $I$ be any solution of (3.8). As $I-I_{w}^{*}$ verifies the system

$$
\begin{cases}\left(I-I_{w}^{*}\right)^{\prime}=-w\left(I-I_{w}^{*}\right), & t \neq(n+l-1) T, t \neq n T \\ \Delta\left(I-I_{w}^{*}\right)=-\delta_{2}\left(I-I_{w}^{*}\right), & t=(n+l-1) T \\ \Delta\left(I-I_{w}^{*}\right)=0, & t=n T\end{cases}
$$

it is seen that

$$
I(t)= \begin{cases}\mathrm{e}^{-w(t-(n-1) T)}\left(I_{0}-\frac{\mu}{1-\mathrm{e}^{-w T}\left(1-\delta_{2}\right)}\right)\left(1-\delta_{2}\right)^{n-1}+I_{w}^{*}(t) ; \quad t \in((n-1) T,(n+l-1) T] \\ \mathrm{e}^{-w(t-(n-1) T)}\left(I_{0}-\frac{\mu}{1-\mathrm{e}^{-w T}\left(1-\delta_{2}\right)}\right)\left(1-\delta_{2}\right)^{n}+I_{w}^{*}(t), \quad t \in((n+l-1) T, n T]\end{cases}
$$

and it follows that for any solution $I(t)$ of (3.8), $\lim _{t \rightarrow \infty}\left|I(t)-I_{w}^{*}(t)\right|=0$. The estimation sought for at the second point can be proved by direct computation.

\section{The extinction of the susceptible pest population}

In this section, we study the situation in which the susceptible pest population tends to extinction. This situation occurs if a certain condition on the total action of the nonlinear force of infection in a period near the infective pest-only equilibrium is satisfied.

Theorem 4.1. The susceptible pest-eradication solution $\left(0, I_{w}^{*}(t)\right)$ is globally asymptotically stable provided that

$$
\int_{0}^{T} g\left(I_{w}^{*}(s)\right) \mathrm{d} s>r T+\ln \left(1-\delta_{1}\right) .
$$

Proof. In order to justify the use of (4.10), we first study the local stability of $\left(0, I_{w}^{*}(t)\right)$ by using small amplitude perturbation methods.

Let us denote

$$
\left\{\begin{array}{l}
S(t)=u(t) \\
I(t)=v(t)+I_{w}^{*}(t)
\end{array}\right.
$$

in which $u$ and $v$ are understood to be small amplitude perturbations. Substituting (4.11) into the first two equations of $(\mathrm{S})$, one obtains 


$$
\left\{\begin{array}{l}
u^{\prime}(t)=u(t) n(u(t))-g\left(v(t)+I_{w}^{*}(t)\right) u(t) \\
v^{\prime}(t)=g\left(v(t)+I_{w}^{*}(t)\right) u(t)-w v(t)
\end{array}\right.
$$

The corresponding linearization of $(4.12)$ at $(0,0)$ is

$$
\left\{\begin{array}{l}
u^{\prime}(t)=r u(t)-g\left(I_{w}^{*}(t)\right) u(t) \\
v^{\prime}(t)=g\left(I_{w}^{*}(t)\right) u(t)-w v(t)
\end{array}\right.
$$

and so a fundamental matrix of (4.13) is

$$
\Phi_{L}(t)=\left(\begin{array}{cc}
\mathrm{e}^{\int_{0}^{t}\left[r-g\left(I_{w}^{*}(s)\right)\right] \mathrm{d} s} & 0 \\
\int_{0}^{t} \mathrm{e}^{-w(t-s)} \mathrm{e}_{0}^{\int_{0}^{s}\left(r-g\left(I_{w}^{*}(\tau)\right)\right) \mathrm{d} \tau} g\left(I_{w}^{*}(s)\right) \mathrm{d} s & \mathrm{e}^{-w t}
\end{array}\right) .
$$

The linearization of the jump conditions at $(n+l-1) T$ reads as

$$
\left\{\begin{array}{l}
\Delta u=-\delta_{1} u(t), \quad t=(n+l-1) T \\
\Delta v=-\delta_{2} v(t)
\end{array}\right.
$$

while the linearization of the jump conditions at $n T$ reads as

$$
\left\{\begin{array}{l}
\Delta u=0, \quad t=n T \\
\Delta v=0
\end{array}\right.
$$

Consequently, the local stability of the susceptible pest-eradication solution $\left(0, I_{w}^{*}(t)\right)$ can be analyzed by studying the eigenvalues of the monodromy matrix

$$
M_{1}=\left(\begin{array}{cc}
1-\delta_{1} & 0 \\
0 & 1-\delta_{2}
\end{array}\right) \Phi_{L}(T)
$$

As the eigenvalues of $M_{1}$ are

$$
\lambda_{1}=\left(1-\delta_{1}\right) \mathrm{e}^{\int_{0}^{T}\left[r-g\left(I_{w}^{*}(s)\right)\right] \mathrm{d} s}, \quad \lambda_{2}=\left(1-\delta_{2}\right) \mathrm{e}^{-w T}
$$

and $0<\lambda_{2}<1$, it follows by Lemma 3.4 that $\left(0, I_{w}^{*}(t)\right)$ is locally asymptotically stable provided that

$$
\left(1-\delta_{1}\right) \mathrm{e}^{\int_{0}^{T}\left[r-g\left(I_{w}^{*}(s)\right)\right] \mathrm{d} s}<1
$$

that is, condition (4.10) holds. We now prove that $\left(0, I_{w}^{*}(t)\right)$ is actually globally asymptotically stable provided that condition (4.10) is satisfied. We first show that $S(t) \rightarrow 0$ as $t \rightarrow \infty$. To this purpose, choose $\varepsilon_{1}>0$ such that

$$
\int_{0}^{T} g\left(I_{w}^{*}(s)-\varepsilon_{1}\right) \mathrm{d} s>r T+\ln \left(1-\delta_{1}\right)
$$

(note that such a choice is feasible, as $\left.\left|g\left(I_{w}^{*}(s)-\varepsilon_{1}\right)-g\left(I_{w}^{*}(s)\right)\right| \leqslant L \varepsilon_{1}\right)$. Let us also denote

$$
\xi=\left(1-\delta_{1}\right) \mathrm{e}^{r T-\int_{0}^{T} g\left(I_{w}^{*}(s)-\varepsilon_{1}\right) \mathrm{d} s}
$$

and observe that $0<\xi<1$. It is seen that

$$
I^{\prime}(t)=g(I(t)) S(t)-w I(t) \geqslant-w I(t)
$$

and so, by Lemma 3.1, I(t) $\geqslant \widetilde{I}(t)$, where $\widetilde{I}(t)$ is the solution of (3.8) with the same initial data at $0+$ as $I$. As any such solution becomes close to $I_{w}^{*}(t)$ for $t \rightarrow \infty$, by Lemma 3.5, there is some $T_{1}>0$ such that $I(t) \geqslant I_{w}^{*}(t)-\varepsilon_{1}$ for $t \geqslant T_{1}$. For the sake of simplicity, we suppose that $I(t)>I_{w}^{*}(t)-\varepsilon_{1}$ for all $t>0$. One then obtains that

$$
\begin{aligned}
S^{\prime}(t) & =S(t) n(S(t))-S(t) g(I(t)) \\
& \leqslant S(t)\left[n(S(t))-g\left(I_{w}^{*}(t)-\varepsilon_{1}\right)\right], \quad t \neq(n+l-1) T
\end{aligned}
$$


and it consequently follows that

$$
\frac{S^{\prime}(t)}{S(t)} \leqslant n(S(t))-g\left(I_{w}^{*}(t)-\varepsilon_{1}\right), \quad t \neq(n+l-1) T .
$$

By integrating the above inequality on $((n+l-1) T,(n+l) T]$, one obtains

$$
\ln S((n+l) T)-\ln S((n+l-1) T+) \leqslant \int_{(n+l-1) T}^{(n+l) T}\left[n(S(s))-g\left(I_{w}^{*}(s)-\varepsilon_{1}\right)\right] \mathrm{d} s
$$

and so

$$
\ln S((n+l) T)-\ln S((n+l-1) T)-\ln \left(1-\delta_{1}\right) \leqslant \int_{(n+l-1) T}^{(n+l) T}\left[r-g\left(I_{w}^{*}(s)-\varepsilon_{1}\right)\right] \mathrm{d} s .
$$

It then follows that

$$
S((n+l) T) \leqslant S((n+l-1) T) \xi
$$

and consequently

$$
S((n+l) T) \leqslant S(l T) \xi^{n}
$$

which implies that $S((n+l) T) \rightarrow 0$ as $n \rightarrow \infty$. Also,

$$
\frac{S^{\prime}(t)}{S(t)}=n(S(t))-g(I(t)) \leqslant r
$$

so

$$
S(t) \leqslant S((n+l-1) T+) \mathrm{e}^{r(t-(n+l-1) T)}, \quad t \in((n+l-1) T,(n+l) T]
$$

which implies that

$$
S(t) \leqslant\left(1-\delta_{1}\right) S((n+l-1) T) \mathrm{e}^{r T}, \quad t \in((n+l-1) T,(n+l) T]
$$

and consequently $S(t) \rightarrow 0$ as $t \rightarrow \infty$. We now prove that $I(t) \rightarrow I_{w}^{*}(t)$ as $t \rightarrow \infty$. To this purpose, let $0<\varepsilon_{2}<w / L$. Since $S(t) \rightarrow 0$ as $t \rightarrow \infty$, there is $T_{2}>0$ such that $S(t)<\varepsilon_{2}$ for $t \geqslant T_{2}$. For the sake of simplicity, we suppose that $S(t)<\varepsilon_{2}$ for all $t>0$. Since

$$
I^{\prime}(t)=g(I(t)) S(t)-w I(t), \quad t \neq(n+l-1) T, \quad t \neq n T
$$

and $g(x) \leqslant L x$ for $x \geqslant 0$, it follows that

$$
-w I(t) \leqslant I^{\prime}(t) \leqslant-\left(w-\varepsilon_{2} L\right) I(t), \quad t \neq(n+l-1) T, \quad t \neq n T .
$$

By Lemma 3.1, it follows that

$$
\widetilde{I}_{1}(t) \leqslant I(t) \leqslant \widetilde{I}_{2}(t)
$$

where $\widetilde{I}_{1}$ and $\widetilde{I}_{2}$ are the solution of (3.8) with the same initial data at $0+$ as $I$ and the solution of (3.8) with $w$ changed into $w-\varepsilon_{2} L$ and the same initial data at $0+$, respectively. As these solutions become close to $I_{w}^{*}(t)$, respectively, to $I_{w-\varepsilon_{2} L}^{*}(t)$, it follows that, for $t$ large enough,

$$
I_{w}^{*}(t)-\varepsilon_{2}<I(t)<I_{w-\varepsilon_{2} L}^{*}(t)+\varepsilon_{2}
$$

and the conclusion now follows from Lemma 3.5.

Note that from (4.10) it follows that the susceptible pest eradication solution is globally asymptotically stable whenever $\delta_{1}>1-\mathrm{e}^{-r T}$, that is, the global asymptotic stability of this solution can be achieved by controlling $\delta_{1}$ alone, which is a natural result (repeatedly retiring enough many susceptible individuals will make the susceptible pest eradication solution globally asymptotically stable). The same result can be achieved provided that $T$ is small enough, but depending on the value of $\delta_{1}$, that is, for $T<(1 / r) \ln \left(1 /\left(1-\delta_{1}\right)\right)$.

By (3.8) and (3.9), it is noted that $\lim _{\mu \rightarrow \infty} \int_{0}^{T} g\left(I_{w}^{*}(s)\right) \mathrm{d} s=+\infty$ for large classes of functions $g$, so the impulsive control is also successful provided that $\mu$ is large enough. The impulsive control is then, theoretically 
speaking, always successful, provided that it is applied often enough ( $T$ is small), enough many susceptible pests die due to pesticide spraying ( $\delta_{1}$ is large) or if enough many infective pests are released periodically ( $\mu$ is large). However, in practical contexts, $\mu$ cannot be arbitrarily large, and $T$ can be limited by other coordinates of human activity, as not enough active time can be sometimes dedicated to pesticide spraying alone. Still, as noted in Section 1, the purpose of the IPM is actually to drive the size of the susceptible pest population below the EIL or the AIL (the infective pests do not count here, as they are assumed not to damage crops), rather than to eradicate the pests completely, so the controls may be successful even if (4.10) is not satisfied, provided that the size of the susceptible pest population stabilizes under the EIL (or AIL).

It is perhaps also worth noting that if $g(x)=x$ for $x \geqslant 0$, then (4.10) reduces to

$$
\mu>\frac{w\left(r T+\ln \left(1-\delta_{1}\right)\right)\left(1-\mathrm{e}^{-w T}\left(1-\delta_{2}\right)\right)}{1-\delta_{2} \mathrm{e}^{-w l T}-\mathrm{e}^{-w T}\left(1-\delta_{2}\right)} .
$$

Combined with a similar rewrite of (5.19) in the following section, this establishes the existence of a threshold parameter for the stability of the system, denoted $\mu_{c}$ and equal to the right-hand side of (4.18). That is, if $\mu>\mu_{c}$ then the susceptible pest-eradication solution is globally asymptotically stable, while if $\mu<\mu_{c}$, then susceptible pest-eradication solution loses its stability and (S) becomes uniformly persistent.

\section{The permanence of the system}

In this section, we study the permanence of the system. To this purpose, we need now introduce the following definition.

Definition 5.1. The system (S) is said to be permanent (uniformly persistent) if there are $m, M>0$ such that for each solution with positive initial data $(S(0), I(0))$, one has $m \leqslant S(t), I(t) \leqslant M$, for enough large $t$.

Of course, if the system (S) is permanent, then both the susceptible and infective pest classes persist in time and minimal numerical levels of subsistence which do not depend on the initial population sizes are assured for each class.

We now prove that the system $(\mathrm{S})$ is permanent provided that the reverse of (4.10) holds.

Theorem 5.1. The system $(\mathrm{S})$ is permanent provided that

$$
\int_{0}^{T} g\left(I_{w}^{*}(s)\right) \mathrm{d} s<r T+\ln \left(1-\delta_{1}\right) .
$$

Proof. It has already been shown that, given $\varepsilon>0$, one may find $T_{\varepsilon}>0$ such that $I(t)>I_{w}^{*}(t)-\varepsilon$ for all $t>T_{\varepsilon}$. Now, it is enough to choose $\varepsilon<\frac{\mu \mathrm{e}^{-w T}\left(1-\delta_{2}\right)}{1-\mathrm{e}^{-w T}\left(1-\delta_{2}\right)}$ and observe that in this situation one has

$$
I(t)>\frac{\mu \mathrm{e}^{-w T}\left(1-\delta_{2}\right)}{1-\mathrm{e}^{-w T}\left(1-\delta_{2}\right)}-\varepsilon \text { for all } t>T_{\varepsilon} .
$$

For the sake of simplicity, let us suppose that the above estimation is satisfied for all $t>0$.

Also, we know that $S$ and $I$ are bounded, by Lemma 3.3. It now remains to prove that $S(t) \geqslant m_{1}$ for some $m_{1}>0$ and $t$ large enough.

First, let $m_{3}>0$ and $\varepsilon_{1}>0$ be small enough, so that

$$
m_{3}<\frac{w}{L}, \quad n\left(m_{3}\right) T+\ln \left(1-\delta_{1}\right)>\int_{0}^{T} g\left(I_{w-m_{3} L}^{*}(t)+\varepsilon_{1}\right) \mathrm{d} t .
$$

As a first step, we now show that one cannot have $S(t)<m_{3}$ for all $t>0$.

We argue by contradiction. Suppose that $S(t)<m_{3}$ for all $t>0$.

Then

$$
I^{\prime}(t)=g(I(t)) S(t)-w I(t) \leqslant-\left(w-L m_{3}\right) I(t), \quad t \neq(n+l-1) T, \quad t \neq n T .
$$


By Lemma 3.1, it follows that $I(t) \leqslant \widetilde{I}_{1}(t)$, where $\widetilde{I}_{1}(t)$ is the solution of (3.8) with the same initial data at $0+$ as $I$ and $w$ changed into $w-L m_{3}$. As this solution becomes close to $I_{w-L m_{3}}^{*}(t)$ as $t \rightarrow \infty$, it follows that there is $T_{1}>0$ such that $I(t) \leqslant I_{w-L m_{3}}^{*}+\varepsilon_{1}$ for $t \geqslant T_{1}$.

Let $n$ large enough, so that $(n+l-1) T>T_{1}$. One then gets

$$
S^{\prime}(t) \geqslant S(t)\left[n\left(m_{3}\right)-g\left(I_{w-m_{3} L}^{*}(t)+\varepsilon_{1}\right)\right], \quad t \neq(n+l-1) T, \quad t \neq n T, \quad t \geqslant T_{1} .
$$

By integrating the above inequality on $((n+l-1) T,(n+l) T]$, one obtains

$$
\ln S((n+l) T)-\ln S((n+l-1) T+) \geqslant n\left(m_{3}\right) T-\int_{(n+l-1) T}^{(n+l) T} g\left(I_{w-m_{3} L}^{*}(t)+\varepsilon_{1}\right) \mathrm{d} t
$$

and so

$$
\ln S((n+l) T)-\ln S((n+l-1) T)-\ln \left(1-\delta_{1}\right) \geqslant n\left(m_{3}\right) T-\int_{(n+l-1) T}^{(n+l) T} g\left(I_{w-m_{3} L}^{*}(t)+\varepsilon_{1}\right) \mathrm{d} t .
$$

Let us denote

$$
\eta=\left(1-\delta_{1}\right) \mathrm{e}^{n\left(m_{3}\right) T-\int_{(n+l-1) T}^{(n+l) T} g\left(I_{w-m_{3} L}^{*}(t)+\varepsilon_{1}\right) \mathrm{d} t}
$$

and observe that, by (5.20), $\eta>1$. It then follows that

$$
S((n+l) T) \geqslant S((n+l-1) T) \eta
$$

and consequently

$$
S((n+l) T) \geqslant S(l T) \eta^{n},
$$

which implies that $S((n+l) T) \rightarrow \infty$ as $n \rightarrow \infty$, which contradicts the boundedness of $S$. It is then seen that one cannot have $S(t)<m_{3}$ for all $t>0$ and consequently there is $t_{1}>0$ such that $S\left(t_{1}\right) \geqslant m_{3}$.

If $S\left(t_{1}\right) \geqslant m_{3}$ for all $t \geqslant t_{1}$, then (S) is persistent and there is nothing left to prove. Otherwise, $S(t)<m_{3}$ for some $t \geqslant t_{1}$. Let us denote

$$
t^{*}=\inf \left\{t>t_{1} ; S(t)<m_{3}\right\} .
$$

To continue our investigation, we need to distinguish whether or not $t^{*}=(n+l-1) T$ for some $n$, so that we could discuss the value of $S\left(t^{*}\right)$. Note that the discussion has to be made in terms of whether or not $t^{*}=(n+l-1) T$ for some $n$ and not in terms of whether or not $t^{*}=n T$ for some $n$, as the jumps of $S$ occur only at $t=(n+l-1) T$.

Case A, $t^{*}=\left(n_{1}+l-1\right) T$ for some $n_{1} \in \mathbb{N}^{*}$.

In this situation, $S(t) \geqslant m_{3}$ for $t \in\left[t_{1}, t^{*}\right]$ and therefore

$$
S\left(t^{*}+\right)=\left(1-\delta_{1}\right) S\left(t^{*}\right) \geqslant\left(1-\delta_{1}\right) m_{3}
$$

and also

$$
S\left(t^{*}+\right) \leqslant m_{3} .
$$

Choose $n_{2}, n_{3} \in \mathbb{N}^{*}$ so that

$$
\begin{aligned}
& \left(n_{2}-1\right) T>\frac{1}{-w+L m_{3}} \ln \frac{\varepsilon_{1}}{M+\frac{\mu}{1-\mathrm{e}^{-\left(w-L m_{3}\right)}}} \\
& \left(1-\delta_{1}\right)^{n_{2}} \mathrm{e}^{n_{2} \eta_{1} T} \eta^{n_{3}}>1,
\end{aligned}
$$

where $\eta$ is given by (5.22) and $\eta_{1}$ is defined as

$$
\eta_{1}=n\left(m_{3}\right)-L M<0 .
$$

Note that $n_{2}, n_{3}$ do not depend on $t^{*}$.

We now show that there should be $t_{2} \in\left(t^{*}, t^{*}+n_{2} T+n_{3} T\right]$ such that $S\left(t_{2}\right)>m_{3}$. Suppose that this is not the case. Then $I(t) \leqslant \widetilde{I}(t)$ on $\left(t^{*}, t^{*}+n_{2} T+n_{3} T\right]$, where $\widetilde{I}$ is the solution of (3.8) with the same initial data at $t^{*}+$ as $I$ and $w$ changed into $w-L m_{3}$. 
In the similar way to the derivation of (3.9), it is seen that

$$
\widetilde{I}(t)=\left\{\begin{array}{lll}
\mathrm{e}^{-\left(w-m_{3} L\right)\left(t-n_{1} T\right)}\left[\widetilde{I}\left(n_{1} T+\right)-\frac{\mu}{1-\left(1-\delta_{2}\right) \mathrm{e}^{-\left(w-m_{3} L\right) T}}\right] & \left(1-\delta_{2}\right)^{n-\left(n_{1}+1\right)}+I_{w-m_{3} L}^{*}(t), & t \in((n-1) T,(n+l-1) T] ; \\
\mathrm{e}^{-\left(w-m_{3} L\right)\left(t-n_{1} T\right)}\left[\widetilde{I}\left(n_{1} T+\right)-\frac{\mu}{1-\left(1-\delta_{2}\right) \mathrm{e}^{-\left(w-m_{3} L\right) T}}\right] & \left(1-\delta_{2}\right)^{n-n_{1}}+I_{w-m_{3} L}^{*}(t), & t \in((n+l-1) T, n T],
\end{array}\right.
$$

for $n \geqslant n_{1}+1$. By the above relations, it follows that

$$
\left|\widetilde{I}(t)-I_{w-m_{3} L}^{*}(t)\right|<\mathrm{e}^{-\left(w-m_{3} L\right)\left(t-n_{1} T\right)}\left|\widetilde{I}\left(n_{1} T+\right)-\frac{\mu}{1-\left(1-\delta_{2}\right) \mathrm{e}^{-\left(w-m_{3} L\right) T}}\right|
$$

for $t>(n+l-1) T, n \geqslant n_{1}+1$.

Also, since

$$
\widetilde{I}\left(n_{1} T+\right)=\mathrm{e}^{-\left(w-m_{3} L\right)\left(n_{1} T-t^{*}\right)} \widetilde{I}\left(t^{*}+\right)+\mu,
$$

one has that

$$
\begin{aligned}
\left|\widetilde{I}\left(n_{1} T+\right)-\frac{\mu}{1-\left(1-\delta_{2}\right) \mathrm{e}^{-\left(w-m_{3} L\right) T}}\right| & =\left|\mathrm{e}^{-\left(w-m_{3} L\right)(1-l) T} I\left(t^{*}+\right)+\mu-\frac{\mu}{1-\left(1-\delta_{2}\right) \mathrm{e}^{-\left(w-m_{3} L\right) T}}\right| \\
& \leqslant M+\frac{\mu}{1-\mathrm{e}^{-\left(w-m_{3} L\right) T}} .
\end{aligned}
$$

For $t \geqslant n_{1} T+\left(n_{2}-1\right) T$, it follows that

$$
\left|\widetilde{I}(t)-I_{w-m_{3} L}^{*}(t)\right| \leqslant \mathrm{e}^{-\left(w-m_{3} L\right)\left(n_{2}-1\right) T}\left(M+\frac{\mu}{1-\mathrm{e}^{-\left(w-m_{3} L\right) T}}\right),
$$

by (5.24), (5.27) and (5.28). By (5.29), it is then seen that

$$
I(t) \leqslant I_{w-m_{3} L}^{*}(t)+\varepsilon_{1}, \quad \text { for } n_{1} T+\left(n_{2}-1\right) T \leqslant t \leqslant t^{*}+n_{2} T+n_{3} T
$$

and therefore (5.21) holds for $n_{1} T+\left(n_{2}-1\right) T \leqslant t \leqslant t^{*}+n_{2} T+n_{3} T$. As a result, by the same argument used for the derivation of (5.23), we deduce that

$$
S\left(t^{*}+n_{2} T+n_{3} T\right) \geqslant S\left(t^{*}+n_{2} T\right) \eta^{n_{3}}
$$

where $\eta$ is given by (5.22).

Since $g(I(t)) \leqslant L I(t) \leqslant L M$, one obtains that

$$
\begin{cases}S^{\prime}(t) \geqslant S(t)\left[n\left(m_{3}\right)-L M\right], & t \neq(n+l-1) T \\ S(t+)=\left(1-\delta_{1}\right) S(t), & t=(n+l-1) T\end{cases}
$$

for $t \in\left[t^{*}, t^{*}+n_{2} T\right]$. Integrating the inequality in (5.31) over $\left(t^{*}, t^{*}+n_{2} T\right]$, one obtains that

$$
S\left(t^{*}+n_{2} T\right) \geqslant m_{3}\left(1-\delta_{1}\right)^{n_{2}} \mathrm{e}^{n_{2} \eta_{1} T} .
$$

By (5.30) and (5.32), one may deduce that

$$
S\left(t^{*}+n_{2} T+n_{3} T\right) \geqslant m_{3}\left(1-\delta_{1}\right)^{n_{2}} \mathrm{e}^{n_{2} \eta_{1} T} \eta^{n_{3}} .
$$

By (5.25), one obtains that $S\left(t^{*}+n_{2} T+n_{3} T\right)>m_{3}$, which is a contradiction, as it was supposed that $S(t) \leqslant m_{3}$ on $\left(t^{*}, t^{*}+n_{2} T+n_{3} T\right]$. It then follows that there is $t_{1} \in\left(t^{*}, t^{*}+n_{2} T+n_{3} T\right]$ for which $S\left(t_{1}\right)>m_{3}$. Let us denote $\tilde{t}_{1}^{*}=\inf _{t>t^{*}}\left\{S(t)>m_{3}\right\}$. Obviously, $\tilde{t}_{1}^{*} \leqslant t^{*}+n_{2} T+n_{3} T$. Also, $S(t) \leqslant m_{3}$ for $t \in\left(t^{*}, \tilde{t}_{1}^{*}\right)$, while $S\left(\tilde{t}_{1}^{*}\right) \geqslant m_{3}$.

As in the derivation of (5.32), one may find that

$$
S(t) \geqslant m_{3}\left(1-\delta_{1}\right)^{n_{2}+n_{3}} \mathrm{e}^{\left(n_{2}+n_{3}\right) \eta_{1} T}, \quad t \in\left(t^{*}, \tilde{t}_{1}^{*}\right)
$$

as $\tilde{t}_{1}^{*} \leqslant\left(n_{2}+n_{3}\right) T$, and so, if we denote,

$$
m_{3}^{\prime}=m_{3}\left(1-\delta_{1}\right)^{n_{2}+n_{3}} \mathrm{e}^{\left(n_{2}+n_{3}\right) \eta_{1} T}
$$


we see that $S(t) \geqslant m_{3}^{\prime}$ for $t \in\left(t^{*}, \tilde{t}_{1}^{*}\right)$ and so $m_{3}^{\prime}$ may be taken as a persistency constant for $S$ on $\left(t^{*}, \tilde{t}_{1}^{*}\right)$. As $S\left(\tilde{t}_{1}^{*}\right) \geqslant m_{3}$, our argument may be continued in the same manner.

Case $\mathrm{B} t^{*} \neq(n+l-1) T$ for all $n \in \mathbb{N}^{*}$.

In this situation, $S$ is continuous at $t^{*}$ and $S(t) \geqslant m_{3}$ for $t \in\left[t_{1}, t^{*}\right]$, while $S\left(t^{*}\right)=m_{3}$, as $t^{*}$ is not a jump point for $S$. Suppose that $t^{*} \in\left(\left(n_{2}+l-1\right) T,\left(n_{2}+l\right) T\right)$ for some $n_{2} \in \mathbb{N}^{*}$.

If $S(t) \leqslant m_{3}$ on the whole interval $\left(t^{*},\left(n_{2}+l\right) T\right]$, then one may continue exactly as in Case A; we omit the details. If there is $t \in\left(t^{*},\left(n_{2}+l\right) T\right)$ such that $S(t)>m_{3}$, then let us denote $\tilde{t}_{2}^{*}=\inf _{t>t^{*}}\left\{S(t)>m_{3}\right\}$. Obviously, $\tilde{t}_{2}^{*} \in\left(t^{*},\left(n_{2}+l\right) T\right], S(t) \leqslant m_{3}$ for $t \in\left(t^{*}, \tilde{t}_{2}^{*}\right)$ and $S\left(\tilde{t}_{2}^{*}\right)=m_{3}$ by continuity. We now study the persistency constant of $S$ on $\left(t^{*}, \tilde{t}_{2}^{*}\right)$.

Since $S(t) \leqslant m_{3}$ on $\left(t^{*}, \tilde{t}_{2}^{*}\right)$, it is seen that

$$
\begin{cases}S^{\prime}(t) \geqslant S(t)\left[n\left(m_{3}\right)-L M\right], & t \neq(n+l-1) T ; \\ S(t+)=\left(1-\delta_{1}\right) S(t), & t=(n+l-1) T,\end{cases}
$$

for $t \in\left(t^{*}, \tilde{t}_{2}^{*}\right)$. Integrating (5.33), we obtain that

$$
S(t) \geqslant S\left(t^{*}\right) \mathrm{e}^{\eta_{1}\left(t-t^{*}\right)} \geqslant m_{3} \mathrm{e}^{\eta_{1} T}
$$

and so, if we denote

$$
m_{4}^{\prime}=m_{3} \mathrm{e}^{\eta_{1} T}
$$

we see that $S(t) \geqslant m_{4}^{\prime}$ for $t \in\left(t^{*}, \tilde{t}_{2}^{*}\right)$ and so $m_{4}^{\prime}$ may be taken as a persistency constant for $S$ on $\left(t^{*}, \tilde{t}_{2}^{*}\right)$. As $S\left(\tilde{t}_{2}^{*}\right)=m_{3}$, our argument may be continued in the same manner.

We now give an approximative interpretation of (5.19). Let us suppose that $(S(t), I(t))$ approaches $\left(0, I_{w}^{*}(t)\right)$. In this situation, it is seen that $\int_{0}^{T} g\left(I_{w}^{*}(s)\right) \mathrm{d} s$ approximates the total (per-susceptible) loss of susceptibles due to new infections in a period $T$, while $r T$ approximates the total (per-susceptible) number of newborn susceptibles, as $n(0)=r$. Also, $\ln \left(1-\delta_{1}\right)$ is a correction term accounting for the loss of susceptibles due to pesticide spraying. If (5.19) is satisfied, then this inequality prevents $(S(t), I(t))$ from being arbitrarily close to $\left(0, I_{w}^{*}(t)\right)$, as there is a net gain of susceptibles near $\left(0, I_{w}^{*}(t)\right)$, while the opposite of (5.19) makes $\left(0, I_{w}^{*}(t)\right)$ globally asymptotically stable, since there is a net loss of susceptibles near $\left(0, I_{w}^{*}(t)\right)$.

\section{Conclusion}

In this paper, an integrated pest management model involving impulsive and periodic biological and chemical controls is investigated. The biological control consists in the periodic release of infective pests, while the chemical control consists in pest poisoning through the use of pesticides. A nonlinear incidence rate of type $g(I) S$ is used to describe disease transmission, where the nonlinear function $g$ represents the so-called force of infection, or attack rate. By means of Floquet theory for impulsive differential equations, it is seen that if a certain inequality involving the total action of the nonlinear force of infection in a period is satisfied, then the susceptible pest-eradication solution is globally asymptotically stable, while if the opposite of this inequality is satisfied then the susceptible pest-eradication solution loses its stability and the system under consideration becomes uniformly persistent.

It is also observed that when $g$ is a linear function, the above results establish the existence of a threshold parameter $\mu_{c}$ for the stability of the system. A biological interpretation of the persistency condition is provided and, in addition, is observed that, theoretically speaking, the impulsive controls can be always made to succeed if enough resources are invested, although in concrete situations these requirements may not necessarily be feasible.

\section{References}

[1] D. Bainov, P. Simeonov, Impulsive Differential Equations: Periodic Solutions and Applications, Longman, John Wiley, New York, NY, 1993.

[2] W.I. Bajwa, M. Kogan, Compendium of IPM Definitions, 1997. Available Online at $<$ http://ippc.orst.edu/IPMdefinitions/> .

[3] V. Capasso, G. Serio, A generalization of Kermack-McKendrick deterministic epidemic model, Math. Biosci. 42 (1978) $43-61$. 
[4] H.W. Hethcote, P. van den Driessche, Some epidemiological models with nonlinear incidence, J. Math. Biol. 29 (1991) $271-287$.

[5] M.P. Hoffmann, A.C. Frodsham, Natural Enemies of Vegetable Insect Pests, Cooperative Extension, Cornell University, Ithaca, NY, 1993.

[6] A. Korobeinikov, Lyapunov functions and global stability for SIR and SIRS epidemiological models with non-linear transmission, Bull. Math. Biol. 30 (2006) 615-626.

[7] A. Korobeinikov, P.K. Maini, Nonlinear incidence and stability of infectious disease models, Math. Med. Biol. 22 (2005) $113-128$.

[8] B. Liu, L. Chen, Y. Zhang, The dynamics of a prey-dependent consumption model concerning impulsive control strategy, Appl. Math. Comput. 169 (2005) 305-320.

[9] W.M. Liu, H.W. Hethcote, S.A. Levin, Dynamical behavior of epidemiological models with nonlinear incidence rates, J. Math. Biol. 25 (1987) 359-380.

[10] W.M. Liu, S.A. Levin, Y. Iwasa, Influence of nonlinear incidence rates on the behavior of SIRS epidemiological models, J. Math. Biol. 23 (1986) 187-204.

[11] B. Liu, Y. Zhi, L. Chen, The dynamics of a predator-prey model with Ivlev's functional response concerning integrated pest management, Acta Math. Appl. Sin. Engl. Ser. 20 (2004) 133-146.

[12] L.P. Pedigo, L.G. Higley, The economic injury level concept and environmental quality: a new perspective, Am. Entomol. 38 (1992) $12-21$.

[13] L.P. Pedigo, S.H. Hutchins, L.G. Higley, Economic injury levels in theory and practice, Annu. Rev. Entomol. 31 (1986) $341-368$.

[14] L.P. Pedigo, Entomology and Pest Management, second ed., Prentice Hall Pub., Englewood Cliffs, NJ, 1996.

[15] S. Ruan, W. Wang, Dynamical behavior of an epidemic model with a nonlinear incidence rate, J. Differential Equations 188 (2003) $135-163$.

[16] Z. Song, Z. Xiang, The prey-dependent consumption two-prey one-predator models with stage structure for the predator and impulsive effects, J. Theor. Biol. 242 (2006) 683-698.

[17] V.M. Stern, R.F. Smith, R. van den Bosch, K.S. Hagen, The integrated control concept, Hilgardia 29 (1959) 81-101.

[18] W. Wang, Epidemic models with nonlinear infection forces, Math. Biosci. Eng. 3 (2006) 267-279.

[19] D. Xiao, S. Ruan, Global analysis of an epidemic model with nonmonotone incidence rate, Math. Biosci., in press, doi:10.1016/ j.mbs.2006.09.025.

[20] H. Zhang, L. Chen, Impulsive control strategies for pest control, J. Biol. Sys., in press. 